\title{
Insomnia in COVID-19 Survivors: A Cross Sectional Study Among Healthcare Workers
}

\author{
Nalakath A. Uvais', Shamsudeen Moideen², Bishurul Hafi', \\ Sooraj Rajagopal ${ }^{4}$, V. Maheshwari ${ }^{5}$, and Tasneem Abdul Gafoor ${ }^{5}$ \\ Departments of ${ }^{1}$ Psychiatry, ${ }^{2}$ Internal Medicine, ${ }^{3}$ Dermatology, Iqraa International Hospital and Research Centre, Calicut, Kerala, India \\ ${ }^{4}$ Department of Physical Medicine and Rehabilitation, Government Medical College, Kozhikkode, India \\ ${ }^{5}$ Nandha College of Pharmacy, Erode, India
}

\begin{abstract}
Objective: Coronavirus disease (COVID-19) infection predisposes patients to various psychiatric disorders. Study results indicate significantly higher prevalence of insomnia among patients with COVID-19 during active phase. Acute insomnia during active phase of COVID-19 can persist even after recovery, and can transform into chronic insomnia. There are not many studies exploring insomnia among COVID-19 survivors. Our study aims to explore insomnia and its correlation among healthcare workers with confirmed infection of COVID-19 after recovery. Methods: One hundred and seven COVID-19 survivors participated in the study. The social demographic and clinical information of all participants was collected by a structured self report questionnaire. Insomnia was evaluated using the Insomnia Severity Index. Results: The prevalence of insomnia was $21.5 \%$ among the healthcare workers with confirmed COVID-19 infection after recovery. There were significant positive correlation between the duration of fever and insomnia severity $(0.247, \mathrm{p}=0.01)$. Conclusion: Our study among healthcare workers recovered from COVID-19 provides evidence for high prevalence of insomnia among COVID-19 survivors. The results of this study demands that specific support measures should be planned and implemented to address insomnia among healthcare workers recovered from COVID-19.

Keywords: COVID-19; Insomnia; Survivors; Healthcare workers; India

Received: June 19, 2021 Revised: September 8, 2021 Accepted: September 8, 2021

Corresponding author: Bishurul Hafi, MD, Department of Dermatology, Iqraa International Hospital and Research Centre, Calicut, Kerala, India.

Tel: 91-7085740869, Fax: 91-4952379102, E-mail: bishuru@gmail.com

(c) This is an Open Access article distributed under the terms of the Creative Commons Attribution Non-Commercial License (https://creativecommons.org/licenses/bync/4.0) which permits unrestricted non-commercial use, distribution, and reproduction in any medium, provided the original work is properly cited.
\end{abstract}

\section{INTRODUCTION}

The novel coronavirus disease (COVID-19) was first observed in the Chinese city of Wuhan in late December 2019 [1]. COVID-19 spread rapidly worldwide due to the highly contagious nature of the virus, and the World Health Organization officially declared COVID-19 as a pandemic on March 11, 2020 [1]. COVID-19 impacted India severely, especially during the second wave. India, with a population of more than 1.34 billion, experienced significant problem in controlling the transmission of COVID-19 infection among its population. While writing this report on June 16, 2021, COVID-19 has infected 29,627,763 patients in India and caused 379,551 deaths (https://www.worldometers.info/, Worldometer, June 16, 2021; 11 IST).
The COVID-19 pandemic affected all aspects of human life. The infection control measures such as lockdown, travel restrictions, and physical distancing changed the way people live, abruptly. Associated social, economic, and health consequences lead to significant psychological problems among general population across the globe [2]. Studies suggest that there are a significant increase in the prevalence of anxiety, depression, and insomnia among general population in most of the countries [3,4]. Studies among the healthcare workers also showed significant increase in psychiatric comorbidities compared to pre-pandemic levels $[5,6]$. Those working in frontline with the COVID-19 patients experienced significantly higher psychological stress [5]. While working in the high risk hospital settings with limited resources, many healthcare workers in India got infected with COVID-19. Accord- 
ing to recent estimates, around 700 doctors died due to COVID-19 during the second wave alone [7].

Emerging evidence suggest that patients with COVID-19 infection suffer from significant psychological problems during the active phase of the illness $[8,9]$. The neurotropic nature of the virus, the symptoms of the infection, absence of effective medicines, risk of complications and mortality, and the need for isolation, contributed to the higher incidence of new onset mental health problems among COVID-19 confirmed patients [10]. However, there are only few studies exploring psychological problems of COVID-19 patients after recovery $[11,12]$. A recent study found that $26.45 \%$ of the COVID-19 patients suffer from insomnia after recovery [11]. Considering higher prevalence of insomnia among COVID-19 patients during acute phase of illness, many of them can potentially develop into chronic insomnia after recovery. To the best of our knowledge, there are no studies exploring insomnia among COVID-19 survivors from India till date. In this cross sectional study, we explored the prevalence of insomnia and its correlation among healthcare workers recovered from COVID-19 infection in South India.

\section{METHODS}

\section{Participants}

This was a cross sectional study conducted among healthcare workers of a tertiary care hospital in South India. The study included healthcare workers who tested positive for COVID-19 according to the provisional guidelines of World Health Organization. The data was collected after they were tested negative for COVID-19. The study protocol was approved by the ethical committee of the Iqraa International Hospital and Research Centre (IEC/2021/06/04). All the participants provided informed consent before responding to the study questionnaire.

\section{Design and measurements}

A structured self-report questionnaire was created for conducting this study. The questionnaire included three sections; sociodemographic details, COVID-19 infection related clinical details, and the Insomnia Severity Index (ISI).

The social demographic variables included age, gender, smoking status, status of physical activity, and presence of comorbid illnesses. The COVID-19 infection related clinical details included the following questions; the duration of fever, length of hospital stay, days since discharge, history of myalgia, COVID-19 pneumonia, intensive care unit admission, oxygen therapy, non-invasive ventilation, mechanical ventilation, and presence of persistent symptoms after recovery from COVID-19.

Insomnia was evaluated by the ISI. ISI is a seven-item self-report index which measures the self-reported level of sleep quality and insomnia, including difficulty in falling asleep, maintaining sleep, and waking up early [13]. Each item is scored from 0 to 4 points. The total score ranges from 0 to 28 . Insomnia on the ISI was classified as none $(0-7)$, subthreshold (8-14), moderate
(15-21), or severe (22-28).

\section{Statistical analysis}

The collected data was entered in Excel format, and the database was established by using SPSS 23.0 statistical software for analysis (IBM Corp., Armonk, NY, USA). Continuous variables were expressed as mean \pm standard deviation. Categorical variables were expressed as frequencies and percentages. Continuous variables were compared with the use of the independent-sample ttest, such as duration fever, duration of hospital stay, and days since discharge from the hospital. Spearman correlation was done to analyze correlation between insomnia severity and other continuous variables. Differences were considered as statistically significant if the $\mathrm{p}$ value was $<0.05$

\section{RESULTS}

\section{Demographic and clinical characteristics}

of all participants

We received responses from 107 hospital staff who were tested positive for COVID-19. Majority of the study population (78.5\%) were between the age group of 20-30 years. Sixty-eight participants $(63.6 \%)$ were females. Nine participants $(8.4 \%)$ had premorbid medical illnesses before testing positive for COVID-19. Of the 107 respondents, 54 (50.5\%) were engaged in regular physical activities and 2 (1.9\%) were active smokers.

Sixty-four (59.8\%) of the study participants had myalgic symptoms associated with COVID-19. Three participants were diagnosed with COVID-19 pneumonia, two participants required intensive care unit admission. Oxygen therapy, non-invasive ventilation, and mechanical ventilation were required for only a single participant. Persistent symptoms after recovery were reported by $71.6 \%$ of the participants. Eighteen $(16.8 \%)$ participants had subthreshold insomnia, and five (4.7\%) participants had clinical insomnia. The sociodemographic and clinical details are summarized in Table 1.

\section{Comparison of demographic and clinical characteristics} between the insomnia group and non-insomnia group in the enrolled COVID-19 patients

A total of 23 people (21.5\%) were diagnosed with insomnia based on the ISI score greater than 8 . The results of the inter-group comparison using chi-square test showed that there was no significant difference in the prevalence of insomnia among patients with different age, gender, comorbid illness, smoking status, physical exercise, history of myalgia, pneumonia, ICU admission, and presence of persistent symptoms. There was a significant positive correlation between duration of fever and insomnia severity $(0.247, \mathrm{p}=0.01)$. There was no statistically significant association between insomnia severity and length of hospital stay and days since discharge from the hospital (Table 2). 
Table 1. Sociodemographic and clinical characteristics of the recovered COVID-19 healthcare workers with or without insomnia

\begin{tabular}{|c|c|c|c|c|}
\hline Variable & Number of patients $(\mathrm{n}=107)$ & No insomnia $(\mathrm{n}=84)$ & Insomnia $(\mathrm{n}=23)$ & p-value \\
\hline Age & & & & 0.35 \\
\hline $20-30$ yrs & $84(78.5)$ & 68 & 16 & \\
\hline $31-40$ yrs & $19(17.8)$ & 12 & 7 & \\
\hline $41-50$ yrs & $4(3.7)$ & 4 & 0 & \\
\hline Gender & & & & 0.22 \\
\hline Female & $68(63.6)$ & 52 & 16 & \\
\hline Male & $39(36.4)$ & 32 & 7 & \\
\hline Comorbidities & & & & 0.33 \\
\hline Yes & $9(8.4)$ & 6 & 3 & \\
\hline No & $98(91.6)$ & 78 & 20 & \\
\hline Physical activity & & & & 0.74 \\
\hline Yes & $54(50.5)$ & 44 & 10 & \\
\hline No & $53(49.5)$ & 40 & 13 & \\
\hline Smoking & & & & 0.76 \\
\hline No & $105(98.1)$ & 82 & 23 & \\
\hline Yes & $2(1.9)$ & 2 & 0 & \\
\hline Myalgia & & & & 0.49 \\
\hline Yes & $64(59.8)$ & 48 & 16 & \\
\hline No & $43(40.2)$ & 36 & 7 & \\
\hline Pneumonia & & & & 0.66 \\
\hline Yes & $3(2.8)$ & 3 & 0 & \\
\hline No & $104(97.2)$ & 81 & 23 & \\
\hline ICU admission & & & & 0.76 \\
\hline Yes & $2(1.9)$ & 2 & 0 & \\
\hline No & $105(98.1)$ & 82 & 23 & \\
\hline Persistent symptoms $(\mathrm{n}=102)$ & & & & 0.08 \\
\hline Yes & $73(71.6)$ & 54 & 19 & \\
\hline No & $29(27.1)$ & 26 & 3 & \\
\hline
\end{tabular}

Data are presented as numbers (\%) or numbers. chi-square test

Table 2. COVID-19 related clinical characteristics of the recovered COVID-19 healthcare workers with or without Insomnia

\begin{tabular}{lccc}
\multicolumn{1}{c}{ Variable } & No insomnia & Insomnia & p value \\
Duration of fever (mean) & 1.57 & 2.21 & 0.22 \\
Length of hospital stay (mean) & 8.1 & 6.8 & 0.29 \\
Days since discharge (mean) & 24.35 & 11 & 0.26 \\
\hline
\end{tabular}

independent-sample t-test

\section{DISCUSSION}

This study showed that healthcare workers with confirmed COVID-19 infection had a high prevalence (21.5\%) of insomnia after recovery. To the best of our understanding, there are only few studies exploring insomnia among patients recovered from COVID-19. A recent study from China by Xu et al. [11] explored insomnia among 125 COVID-19 survivors and found that $26.45 \%$ of the participants reported insomnia based on the ISI score greater than 8 . They also found age and presence of physical and mental impairment as risk factors for insomnia among COVID-19 survivors. Another social media based survey study explored insomnia among 1,215 participants from 15 countries us- ing Athena's Insomnia Scale, and found that 943 (77.6\%) participants had a score $\geq 6$ representing insomnia [14]. The authors also found that age, country, and symptoms predict the risk for insomnia among COVID-19 survivors. Our study also found significant positive correlation between symptom (duration of fever) and the severity of insomnia. Another recent retrospective study by Taquet et al. [12] evaluated neuropsychiatric outcome among 236,379 survivors of COVID-19 infection in the 6 months following a COVID-19 diagnosis based on electronic health records. The authors found that $5.4 \%$ of all COVID-19 patients in the study experienced insomnia in the 6 month period following infection, and the prevalence of insomnia significantly increased with severity of COVID-19 infection and the need for hospital care.

The prevalence of insomnia in our study is significantly higher when compared the prevalence of insomnia among general population. A recent meta-analysis of 177 studies comprising 345,270 participants from 39 countries regarding sleep problems during COVID-19 pandemic reported that the corrected pooled estimated prevalence of sleep problems were $18 \%$ among the general population [15]. Another survey study from India among 958 respondents spread across 25 states and from all regions of the coun- 
try reported that around $10 \%$ of the study participants reported clinically significant insomnia according to ISI [16].

The prevalence of insomnia among healthcare workers recovered from COVID-19 is significantly higher when compared to findings of a previous study among healthcare workers from the same hospital during the early phase of COVID-19 pandemic. A cross-sectional study conducted in May, 2020 among 347 hospital staff of our hospital found that only $13.3 \%$ reported insomnia according to ISI [17]. However, the prevalence of insomnia in our study is significantly less when compared to multiple studies among healthcare workers from across the world during COVID-19 pandemic. A recent meta-analysis showed that the corrected pooled estimated prevalence of sleep problems among healthcare workers from 39 countries were $31 \%$ [15]. One of the reason for this higher prevalence could be that many studies included frontline hospital staff who are actively involved in COVID-19 management, and significantly higher rate of insomnia was reported among them. Moreover, the relatively lower prevalence of sleep disorder in our study also can be due to difference in scales used across studies [9]. It has been found that studies using the Pittsburgh Sleep Quality Index (PSQI) scale found significantly higher rate insomnia among the study participants as the questions in the PSQI assess wide range of sleep-related issues [9].

The prevalence of insomnia in our study is significantly higher when compared to the prevalence of insomnia among active COVID-19 patients in the same setting. A recent study from our hospital regarding prevalence of insomnia among 136 COVID-19 patients found that $19.1 \%$ patients had insomnia according to ISI [9].

In conclusion, our study among healthcare workers recovered from COVID-19 provides evidence for high prevalence of insomnia among COVID-19 survivors. The results of this study demands that specific support measures should be planned and implemented to address insomnia among healthcare workers recovered from COVID-19. Moreover, our study results also has research and policy implications. Future well conducted prospective studies are necessary to understand the course of insomnia among COVID-19 survivors.

\section{Acknowledgments}

None

\section{Conflicts of Interest}

The authors have no potential conflicts of interest to disclose.

\section{Author Contributions}

Conceptualization: all authors. Data curation: all authors. Formal analysis: all authors. Funding acquisition: all authors. Investigation: all authors. Methodology: all authors. Project administration: all authors. Resources: all authors. Software: all authors. Supervision: all authors. Validation: all authors. Visualization: all authors. Writing—original draft: all authors. Writing—review \& editing: all authors.

\section{ORCID iDs}

Nalakath A. Uvais (D)

https://orcid.org/0000-0003-0961-3025

Shamsudeen Moideen (D)

https://orcid.org/0000-0002-4362-0424

Bishurul Hafi (D)

https://orcid.org/0000-0001-9749-8906

Sooraj Rajagopal (D)

https://orcid.org/0000-0002-3375-1527

V. Maheshwari (D)

https://orcid.org/0000-0002-6911-4044

Tasneem Abdul Gafoor (D)

https://orcid.org/0000-0003-4363-3855

\section{REFERENCES}

1. Jahrami H, BaHammam AS, Bragazzi NL, Saif Z, Faris M, Vitiello MV. Sleep problems during the COVID-19 pandemic by population: a systematic review and meta-analysis. J Clin Sleep Med 2021;17:299-313.

2. Nochaiwong S, Ruengorn C, Thavorn K, Hutton B, Awiphan R, Phosuya C, et al. Global prevalence of mental health issues among the general population during the coronavirus disease-2019-pandemic: a systematic review and meta-analysis. Sci Rep 2021;11:10173.

3. Salari N, Hosseinian-Far A, Jalali R, Vaisi-Raygani A, Rasoulpoor S, Mohammadi $\mathrm{M}$, et al. Prevalence of stress, anxiety, depression among the general population during the COVID-19 pandemic: a systematic review and metaanalysis. Global Health 202;16:57.

4. Chakraborty K, Chatterjee M. Psychological impact of COVID-19 pandemic on general population in West Bengal: a cross-sectional study. Indian J Psychiatry 2020;62:266-272

5. Pappa S, Ntella V, Giannakas T, Giannakoulis VG, Papoutsi E, Katsaounou P. Prevalence of depression, anxiety, and insomnia among healthcare workers during the COVID-19 pandemic: a systematic review and meta-analysis. Brain Behav Immun 2020;88:901-907.

6. De Kock JH, Latham HA, Leslie SJ, Grindle M, Munoz SA, Ellis L, et al. A rapid review of the impact of COVID-19 on the mental health of healthcare workers: implications for supporting psychological well-being. BMC Public Health 2021;21:104.

7. New Delhi Television. 719 Doctors died due to Covid during second wave: top medical body [Internet]. Available at: https://www.ndtv.com/indianews/coronavirus-719-doctors-died-due-to-covid-during-second-waveindian-medical-association-2462232. Accessed July 12, 2021.

8. Sahoo S, Mehra A, Dua D, Suri V, Malhotra P, Yaddanapudi LN, et al. Psychological experience of patients admitted with SARS-CoV-2 infection. Asian J Psychiatr 2020;54:102355.

9. Uvais NA, Moideen S, Hafi NAB, Rajagopal S, Maheshwari V, Gafoor TA. Insomnia among active patients with COVID-19: a cross-sectional study. Chronobiol Med 2021;3:31-34.

10. Mukaetova-Ladinska EB, Kronenberg G. Psychological and neuropsychiatric implications of COVID-19. Eur Arch Psychiatry Clin Neurosci 2021;271: 235-248.

11. Xu F, Wang X, Yang Y, Zhang K, Shi Y, Xia L, et al. Depression and insomnia in COVID-19 survivors: a cross-sectional survey from Chinese rehabilitation centers in Anhui province. Sleep Med 2021 Feb 8 [Epub]. Available at: https://doi.org/10.1016/j.sleep.2021.02.002.

12. Taquet M, Geddes JR, Husain M, Luciano S, Harrison PJ. 6-month neurological and psychiatric outcomes in 236379 survivors of COVID-19: a retrospective cohort study using electronic health records. Lancet Psychiatry 2021;8:416-427.

13. Morin CM, Belleville G, B?langer L, Ivers H. The Insomnia Severity Index: psychometric indicators to detect insomnia cases and evaluate treatment response. Sleep 2011;34:601-608.

14. Hashim HT, Varney JA, Ramadhan MA, Sarfraz A, Sarfraz Z, Murry K, et al. Insomnia among recovered COVID-19 patients. J Sleep Disord Ther 
2021;10:328.

15. Alimoradi Z, Broström A, Tsang HWH, Griffiths MD, Haghayegh S, Ohayon MM, et al. Sleep problems during COVID-19 pandemic and its' association to psychological distress: a systematic review and meta-analysis. EClinicalMedicine 2021;36:100916.

16. Gupta R, Grover S, Basu A, Krishnan V, Tripathi A, Subramanyam A, et al.
Changes in sleep pattern and sleep quality during COVID-19 lockdown. Indian J Psychiatry 2020;62:370-378

17. Uvais NA, Nalakath MJ, Jose K. Facing COVID-19: psychological impacts on hospital staff in a tertiary care private hospital in India. Prim Care Companion CNS Disord 2021;23:20m02843. 